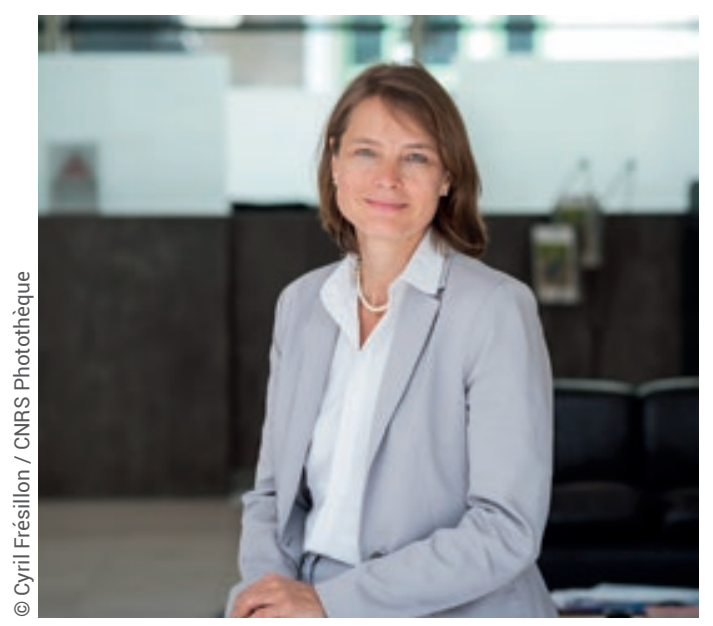

\title{
Astrid Lambrecht, la curiosité en partage
}

\author{
Spécialiste de mécanique quantique, cette théoricienne proche \\ de l'expérience dirige l'Institut de physique (INP) du CNRS \\ depuis juin 2018. Elle y encourage une recherche où I'exploration \\ des fondements de la physique, ses interfaces avec les autres \\ disciplines et l'innovation se nourrissent mutuellement.
}

«Ce n'est pas en améliorant la bougie que l'on a inventé l'ampoule électrique! " Par cette citation attribuée au célèbre physicien Niels Bohr, Astrid Lambrecht, à la tête de l'Institut de physique (INP) du CNRS depuis juin 2018, résume sa conception de la physique, de même que l'esprit de sa politique scientifique. Et la physicienne développe : "La physique a pour but de découvrir les lois fondamentales de notre univers. En même temps, c'est une source inépuisable d'applications, et nombre de ses découvertes ont eu pour origine la réponse à des questions pratiques. " Elle ajoute : "Ce qui doit primer, c'est la curiosité !" Qui, selon cette scientifique d'origine allemande, n'est jamais aussi bien éveillée que lorsque la physique devient cette formidable aventure collective où théoriciens et expérimentateurs travaillent de concert, explorant aussi bien les fondements de leur science que son interface avec les autres disciplines.

Au départ, cette spécialiste reconnue de l'effet Casimir, l'une des manifestations les plus déconcertantes de la mécanique quantique, n'imagine pourtant même pas faire de la physique, et s'inscrit en première année de médecine à l'issue de ses études secondaires. "J'avais plus de goût pour la biologie et les sciences naturelles, plus concrètes, plus proches de la vie quotidienne ", se souvient-elle. Un cours de physique " extraordinaire " en décide autrement : "J'ai découvert avec ravissement cette possibilité offerte par la physique de se poser des questions très fondamentales tout en allant au bout d'une interrogation ", précise Astrid Lambrecht.

Elle opte alors pour une réorientation au sein de l'Université d'Essen, en Allemagne, avant un séjour prédoctoral à l'Imperial College de Londres, en 1991. La future
Médaille d'argent du CNRS y découvre l'optique quantique, alors en pleine révolution, et plus généralement "l'étrange " mécanique quantique et ses problèmes " très ouverts, très difficiles, mais très intéressants!" Après quoi, Astrid Lambrecht, recommandée par ses professeurs, rejoint le Laboratoire Kastler Brossel (LKB) à Paris, où elle effectue sa thèse de doctorat sous la direction d'Élisabeth Giacobino. Après un séjour postdoctoral dans le laboratoire de Theodor Wolfgang Hänsch, prix Nobel de physique 2005, à l'Institut Max Planck, à Garching, elle est recrutée par le CNRS en 1996.

De retour au LKB, son goût pour les questions ardues pousse Astrid Lambrecht à s'intéresser à l'effet Casimir, du nom du physicien qui l'a prédit le premier, en 1948. Selon les calculs de ce dernier, deux plaques conductrices se faisant face exercent l'une sur l'autre une force attractive ayant pour origine ce que les physiciens appellent les "fluctuations du vide" (fig. 1). Celles-ci reposent sur l'idée qu'à l'échelle quantique, le vide n'est pas rien, mais un milieu qui, même en l'absence de toute matière, demeure agité de fluctuations quantiques irréductibles...

L'idée de la physicienne : rendre tangible cette manifestation déroutante de la mécanique quantique. Comme elle l'explique, alors même qu'en ce début des années 2000, l'étude de l'effet Casimir connait un renouveau sur le plan expérimental, "avec Serge Reynaud, nous avons introduit un formalisme issu de l'optique quantique permettant de faire des prédictions dans des situations expérimentales à la fois réalistes et variées, notamment en tenant compte de la réflexion de la lumière forcément imparfaite des miroirs. "
À la suite, la lauréate du Prix Aimé Cotton 2005 de la Société française de physique, en collaboration notamment avec Marc-Thierry Jaekel, poursuit des travaux très fondamentaux sur l'effet Casimir dynamique, selon lequel un miroir se déplaçant très rapidement dans le vide devrait émettre des photons " arrachés " à celui-ci. En parallèle, elle collabore avec des équipes expérimentales sur différents aspects concrets de l'effet Casimir, soit qu'il faille en tenir compte pour interpréter correctement les résultats d'une expérience de physique des particules, soit qu'il ait des implications dans le développement de composants nanotechnologiques.

Car, pour Astrid Lambrecht, la finalité importe peu pourvu qu'il y ait toujours quelque chose de nouveau à apprendre et à partager. Décrivant son activité de chercheuse, elle évoque en effet spontanément l'importance pour elle des discussions avec ses pairs, notamment les doctorantes et doctorants dont elle a eu la charge. De même, celle qui a été directrice adjointe scientifique de l'INP entre 2016 et 2018, possède cette conviction d'appartenir à une communauté, non sans lien avec son choix de s'investir dans l'administration du CNRS. "C'est une façon de rendre à un établissement qui m'a accueilli et tant offert ", précise-t-elle.

Avec l'écoute comme méthode de travail : "La politique scientifique de l'INP s'appuie en premier lieu sur les remontées des laboratoires, détaille la successeuse d'Alain Schuhl, aujourd'hui directeur général délégué à la science du CNRS. Les chercheuses et les chercheurs sont de fait les mieux placés pour savoir où sont les questions intéressantes, les sujets qui comptent ou les problématiques qui montent. " 


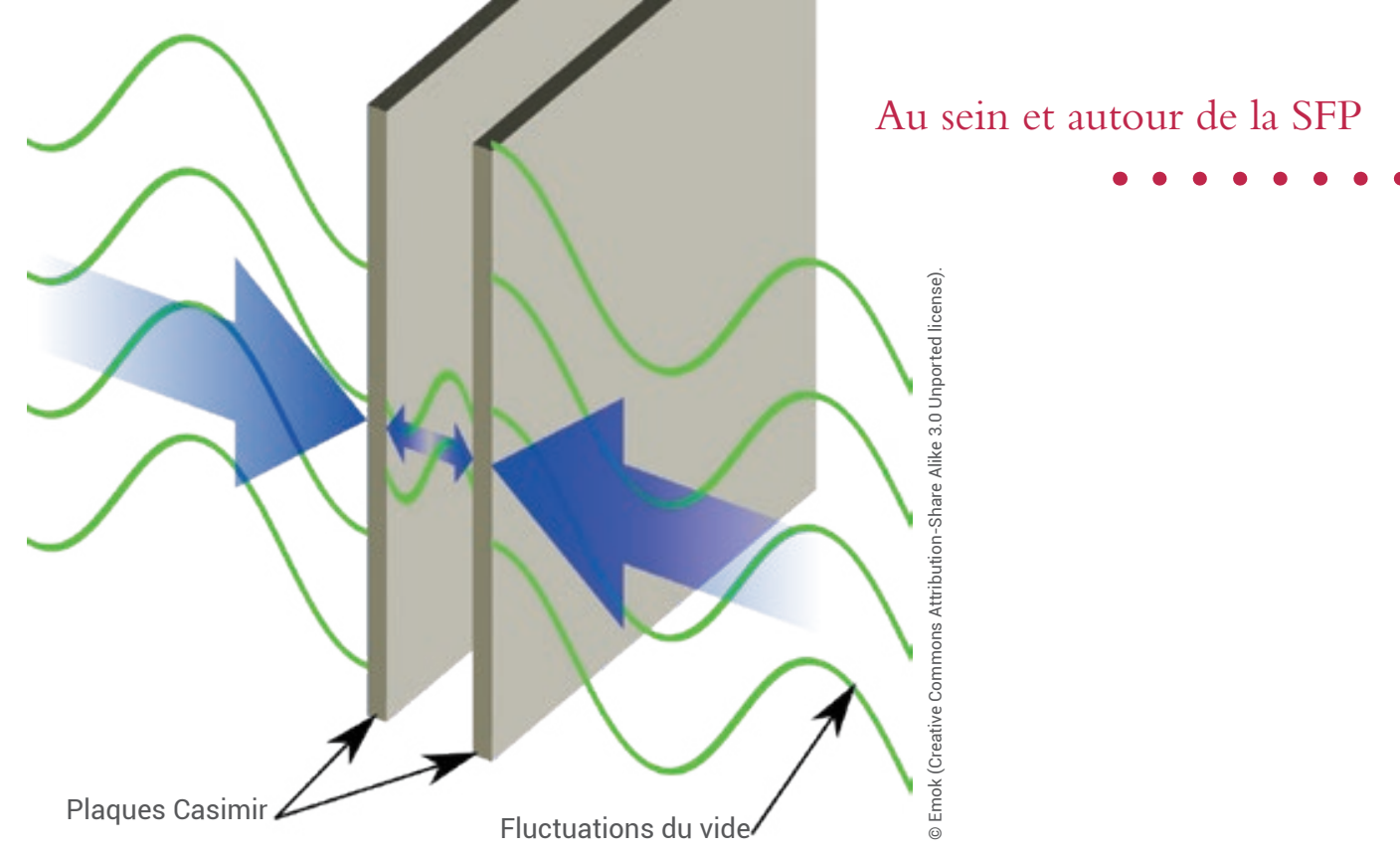

Pour minimiser le risque de passer à côté de quelque chose, Astrid Lambrecht croise ainsi les regards à tous les niveaux, des Groupements de recherche (GdR) au Conseil scientifique de l'institut, ou des Directions adjointes scientifiques au Comité national du CNRS. Concrètement, à son initiative, la fréquence des séminaires regroupant les directrices et directeurs adjoints a été augmentée. Quant aux exercices de prospective des GdR, dont le dernier a fait l'objet d'une synthèse publiée cette année [1], ils seront réactualisés très régulièrement. "Il est important de rechercher la cohérence des visions aux différents étages hiérarchiques, de même que de se donner les moyens d'identifier les signaux faibles ", appuie celle qui est également membre du Conseil scientifique de l'Office parlementaire d'évaluation des choix scientifiques et technologiques.

Consulter, écouter, échanger... mais également décider : "Je suis une pragmatique, et piloter la recherche, cela signifie l'aider à se structurer ", souligne Astrid Lambrecht. C'est pourquoi un chargé de mission a effectué sur le territoire le recensement des microscopes électroniques en transmission : "Il s'agit d'optimiser le déploiement de ces instruments qui sont indispensables, et dont il faut assurer le cout de maintien et de fonctionnement ", explique la directrice.

Pour Astrid Lambrecht, structurer c'est aussi aider à l'émergence de nouvelles communautés. C'est le sens du GdR Polymères et Océans, créé en début d'année. Initié par l'INP, il regroupe des scientifiques de six instituts du CNRS et d'autres partenaires comme l'IFREMER, autour du devenir des matières plastiques dans les eaux marines. De même, à

1. Forces de Casimir sur des plaques parallèles. L'effet Casimir, prédit par le physicien néerlandais Hendrik Casimir en 1948, est une force attractive entre deux plaques parallèles et non chargées. Il est dû aux fluctuations quantiques du vide.

l'échelle internationale, la directrice se félicite de la mise en route, en 2019, d'un laboratoire commun international entre un laboratoire du CNRS, le CEMES, et l'entreprise japonaise Hitachi.

Au-delà, la théoricienne quantique insiste sur le caractère pivot de la physique qui, selon sa conception, est autant une discipline qu'une méthode ou une manière d'interroger le monde. D'où, pour elle et l'INP, l'importance d'encourager une recherche interdisciplinaire, tout en soutenant la recherche disciplinaire en physique, indispensable à la première. À cet égard, la lauréate 2016 du prix Gentner-Kastler, décerné conjointement par la Société française de physique et son homologue allemande, cite l'importance des liens entre physique, biologie et médecine, notamment dans le domaine de l'imagerie. S'agit-il de science fondamentale, de science appliquée? Pour Astrid Lambrecht, la question est presque sans objet, rappelant que la mécanique quantique est à l'origine du laser, aujourd'hui incontournable dans tous les segments de la société, et qu'à l'inverse, Hendrik Casimir a découvert l'effet qui porte son nom en travaillant au développement d'ampoules électriques chez Philips. Sublime illustration de cette dialectique avec, l'année passée, la réforme du Système international d'unités, désormais toutes fondées sur des constantes fondamentales de la physique, et sur lesquelles reposent les certifications métrologiques qui permettent au final d'acheter un kilo de pommes!
Ainsi, pour Astrid Lambrecht, il ne fait aucun doute que l'INP, à l'origine de 80 start-up en activité et qui a reçu cinq grands prix au concours d'innovation i-Lab 2018, doit encourager l'innovation. "À cet égard, nous avons à l'Institut un programme de "coaching" afin d'aider nos chercheurs à franchir le pas, notamment en leur faisant comprendre que la valorisation d'un résultat ne signifie pas mettre leur recherche de côté, mais qu'au contraire, les deux activités sont complémentaires et se nourrissent l'une l'autre ", plaide-t-elle.

In fine, un seul mot d'ordre : l'excellence disciplinaire. Dans ce but, la directrice de l'INP insiste notamment sur l'importance de laisser aux chercheurs le temps nécessaire, parfois long, d'explorer au-delà des limites de notre connaissance. La nécessité de donner parfois un coup de pouce aux scientifiques en finançant des projets novateurs compte tout autant : "C'est le sens des projets dits "Levée de verrous" qui ont permis l'année passée à quinze chercheurs de l'INP d'être accompagnés dans l'exploration de voies nouvelles, inédites dans leur champ disciplinaire. "Telles celles empruntées par Astrid Lambrecht au cours de sa carrière de chercheuse. Seule façon de découvrir, qui sait, un jour, ce qui remplacera peutêtre demain l'ampoule électrique !

Mathieu Grousson, journaliste scientifique

[1] https://inp.cnrs.fr/fr/node/1970 